\title{
Activities of the Primary and Supplementary Motor Areas Increase in Preparation and Execution of Voluntary Muscle Relaxation: An Event-Related fMRI Study
}

\author{
Keiichiro Toma, ${ }^{1}$ Manabu Honda, ${ }^{1}$ Takashi Hanakawa, ${ }^{1}$ Tomohisa Okada, ${ }^{2}$ Hidenao Fukuyama, ${ }^{1}$ Akio Ikeda, ${ }^{1}$ \\ Sadahiko Nishizawa, ${ }^{2}$ Junji Konishi, ${ }^{2}$ and Hiroshi Shibasaki ${ }^{1}$ \\ Departments of ${ }^{1}$ Brain Pathophysiology and ${ }^{2}$ Nuclear Medicine, Kyoto University Graduate School of Medicine, Kyoto, \\ 606-8507 Japan
}

Brain activity associated with voluntary muscle relaxation was examined by applying event-related functional magnetic resonance imaging (fMRI) technique, which enables us to observe change of $\mathrm{fMRI}$ signals associated with a single motor trial. The subject voluntarily relaxed or contracted the right upper limb muscles. Each motor mode had two conditions; one required joint movement, and the other did not. Five axial images covering the primary motor area (M1) and supplementary motor area (SMA) were obtained once every second, using an echoplanar 1.5 tesla MRI scanner. One session consisted of 60 dynamic scans (i.e., $60 \mathrm{sec}$ ). The subject performed a single motor trial (i.e., relaxation or contraction) during one session in his own time. Ten sessions were done for each task. During fMRI scanning, electromyogram (EMG) was monitored from the right forearm muscles to identify the motor onset. We calculated the correlation between the obtained $\mathrm{fMRI}$ signal and the expected hemodynamic response. The muscle relaxation showed transient signal increase time-locked to the EMG offset in the M1 contralateral to the movement and bilateral SMAs, where activation was observed also in the muscle contraction. Activated volume in both the rostral and caudal parts of SMA was significantly larger for the muscle relaxation than for the muscle contraction $(p<0.05)$. The results suggest that voluntary muscle relaxation occurs as a consequence of excitation of corticospinal projection neurons or intracortical inhibitory interneurons, or both, in the M1 and SMA, and both pre-SMA and SMA proper play an important role in motor inhibition.

Key words: voluntary muscle relaxation; voluntary muscle contraction; event-related functional magnetic resonance imaging; primary motor area; pre-supplementary motor area; supplementary motor area proper
In the execution of complex movements in our daily motor repertoire, not only muscle contraction but also muscle relaxation plays an important role. Repetitive or sequential motor tasks, which have been frequently adopted in neuroimaging studies on human motor control (Roland et al., 1980; Shibasaki et al., 1993), contain elements of both muscle contraction and its termination within a motor task, but so far little attention has been paid to the distinction between the two: muscle contraction and relaxation.

Previous monkey experiments demonstrated that the muscle contraction can be inhibited after intracortical microstimulation or spontaneous firing of corticomotoneurons (Jankowska et al., 1976; Cheney et al., 1985; Lemon et al., 1987; Schmidt and McIntosh, 1990), suggesting the presence of neurons within the motor cortex whose activity increases in association with the termination of muscle contraction. Clinically, myoclonic jerk associated with the silent period in electromyogram (EMG) is known as "negative myoclonus" (Young and Shahani, 1986), and some of them were shown to be of cortical origin, namely the

Received Nov. 23, 1998; revised Feb. 10, 1999; accepted Feb. 12, 1999.

This work was supported in part by Grants-in-Aid for Scientific Research (A) 09308031, (A) 08558083, and (C) 08670705, on Priority Areas 08279106, and for International Scientific Research 10044269 from the Japan Ministry of Education, Science, Sports, and Culture, Research for the Future Program JSPSRFTF97L00201 from the Japan Society for the Promotion of Science, and General Research Grant for Aging and Health from the Japan Ministry of Health and Welfare.

Correspondence should be addressed to Dr. Hiroshi Shibasaki, Department of Brain Pathophysiology, Kyoto University Graduate School of Medicine, 54 Kawahara-cho, Shogoin, Sakyo-ku, Kyoto, 606-8507 Japan.

Copyright (C) 1999 Society for Neuroscience $\quad 0270-6474 / 99 / 193527-08 \$ 05.00 / 0$ consequence of abnormal discharge of cortical neurons (Ugawa et al., 1989; Shibasaki et al., 1994; Tassinari et al., 1995). It is thus postulated that the activity of some cortical areas increases in association with motor inhibition. Previously we showed, by backaveraging the scalp-recorded potentials with respect to the EMG offset, that Bereitschaftspotential (BP) occurs before the voluntary muscle relaxation, and it shows the similar scalp topography to that preceding the voluntary muscle contraction (Terada et al., 1995). This finding suggests that almost the same areas may play an active role in both the muscle relaxation and contraction. In this paper, we defined "inhibitory motor area" as cortical regions where the spontaneous neuronal activity or the external stimulation gives rise to the termination of ongoing movement or muscle contraction. It may contain the "negative motor area" proposed by Lüders et al. (1995). Physiologically, motor inhibition might be associated with neuronal discharges of either the projection neurons or intracortical interneurons. Activation of the latter may be detected as an increase in regional cerebral blood flow ( $\mathrm{rCBF}$ ) by using positron emission tomography (PET) or functional magnetic resonance imaging (fMRI), but may not be so by the recording of BP.

The conventional neuroimaging techniques such as PET and block-designed fMRI presuppose a steady state of the changes in rCBF during repetitive execution of the same tasks over a period of several tens of seconds to a few minutes (Roland et al., 1980; Shibasaki et al., 1993). By these techniques, therefore, it is difficult to distinguish the neuronal activity associated with the muscle relaxation from that with muscle contraction because they are 
intermingled in such repetitive motor tasks. By contrast, the more recently developed fMRI technique with echoplanar imaging enables us to map the second by second time course of the hemodynamic response in relation to an event of brief duration with high spatial resolution (Buckner et al., 1996; Rosen et al., 1998), which is termed "event-related fMRI." In the present study, to disclose the neural substrates underlying the motor inhibitory mechanism, we examined the brain activity associated with the preparation and execution of voluntary muscle relaxation in the primary motor area (M1) and supplementary motor area (SMA), using the event-related fMRI technique.

\section{MATERIALS AND METHODS}

Subjects. Eight normal volunteers (seven male and one female; age, 21-34 years; mean age, 29.5) took part in the present study. All were righthanded, and none of them had a previous history of any neurological or psychiatric disorders. All gave informed consent before the experiment.

Behavioral paradigm. The behavioral paradigm was composed of four tasks with a two by two factorial design. Two motor modes, muscle relaxation and muscle contraction, were compared in this study. Each mode had two conditions; one required joint movement (i.e., movement), and the other did not (i.e., isometric). All the tasks were performed with the subject's right hand. For the sake of analysis, each trial was divided into three different phases: "premotor", "motor", and "postmotor" for all the tasks.

In the muscle relaxation mode under movement condition (R_mv), the subject held the right wrist in horizontal plane with palm down by maintaining moderate contraction of the wrist extensor muscles (premotor phase). Then the subject relaxed those muscles as quickly as possible, resulting in abrupt wrist drop after the gravity (motor phase), and kept the relaxed position until the end of the session (postmotor phase).

In the muscle contraction mode under movement condition ( $\left.\mathrm{C}_{-} \mathrm{mv}\right)$, after holding the horizontal position of the right wrist like in the $\mathrm{R}_{-} \mathrm{mv}$, the subject extended the right wrist as quickly as possible up to $\sim 60^{\circ}$ from the horizontal plane and kept the extended position until the end of the session.

In the muscle relaxation mode under isometric condition ( $\mathrm{R}_{-}$iso), the subject pressed the right thumb against the index and middle fingers with moderate contraction of the involved muscles (a pinch). Then the subject suddenly released the force by relaxing those muscles and kept the relaxed state until the end of the session.

In the muscle contraction mode under isometric condition ( $\mathrm{C}_{-}$iso), after holding a pinch with moderate muscle contraction like in the $\mathrm{R}_{-}$iso, the subject made the maximal contraction of the corresponding muscles as quickly as possible and kept the maximal contraction until the end of the session.

Before the image acquisition, the subject was trained to perform all of the four tasks by monitoring surface EMGs until they could keep the EMG activities of other irrelevant muscles silent. Especially for the R_mv, the subject was well trained to drop the right wrist after the gravity without concomitant contraction of the antagonist muscles [e.g., flexor carpi ulnaris muscle (FCU)] at the moment of the muscle relaxation (see also Terada et al., 1995). The subject was also trained to control the amount of EMG activities during the premotor phase nearly identical between the muscle relaxation and contraction modes for each condition.

The subject was laid supine on a bed with earplugs, and his head was immobilized with a forehead strap and urethane foam pads. The subject was instructed to set up the premotor position or posture several seconds before the beginning of each session. For all the tasks, the subject performed a single motor trial in a self-initiated manner at $\sim 25-30 \mathrm{sec}$ after the beginning of the session without verbally counting the timing. During the postmotor phase, the subject was requested to keep the hand position or posture until the end of each session to avoid the contamination by the signals caused by additional movements. Ten sessions were successively performed for each task. For each subject, each of the movement and isometric conditions was performed en block (two motor tasks for the movement condition followed by the two for the isometric condition, or vice versa), and the kind of motor tasks for the initial session was counterbalanced across the subjects.

To identify the timing of the motor trial, surface EMG signals from the bilateral extensor carpi radialis, FCU, and flexor digitorum sublimis muscles were recorded during fMRI scanning, by using a digital EEGEMG system (EEG 2100; Nihonkoden, Tokyo, Japan). A pair of carbon

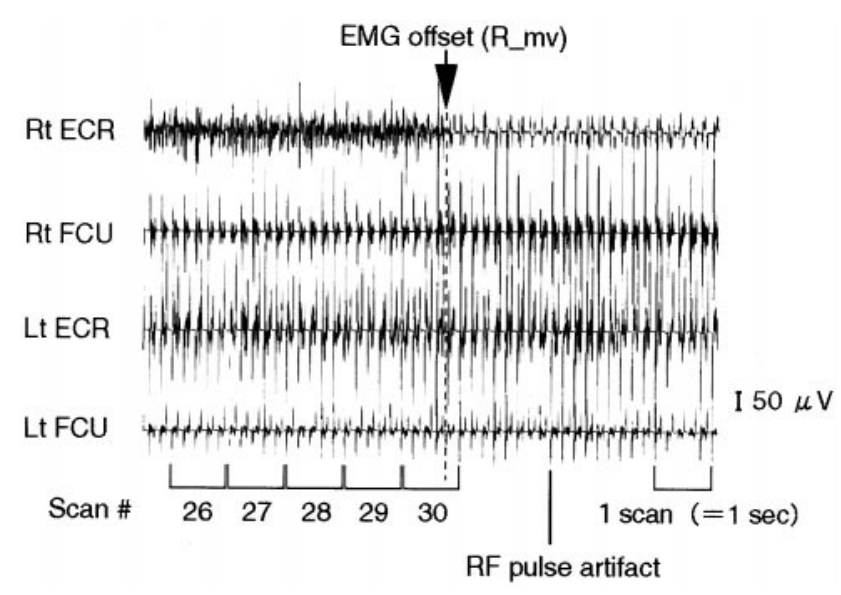

Figure 1. Surface EMGs recorded from the bilateral forearm muscles during fMRI scanning ( $\mathrm{R}$ mv task). Despite the conspicuous signals caused by radio frequency pulses on the record, the EMG offset (arrow) can be identified, which happens to coincide with the 30th scan in this particular session. ECR, Extensor carpi radialis muscle; $F C U$, flexor carpi ulnaris muscle.

electrodes (BRS-150-E; NEC Medical Systems, Tokyo, Japan) were placed over each muscle belly, $2 \mathrm{~cm}$ apart from each other. The EMG signals were filtered with $30-120 \mathrm{~Hz}$ passband $(-3 \mathrm{~dB})$, digitized at 500 $\mathrm{Hz}$, and stored on a magneto-optical disk for the later analysis. During the image acquisition, radio frequency pulses arising from the MR system produced electric signal on the EMG record at a regular pace. By referring to these signals, the timing of the motor trial was identified as an abrupt decrease and increase of EMG discharges for the muscle relaxation and contraction, respectively (Fig. 1). Additionally, two of the authors (K.T. and T.O.) inspected the timing of the motor trial as well as the task performance throughout the image acquisition. After the image acquisition, the subject was asked to verbally report the subjective difficulty of each task.

Image acquisition. Functional imaging was conducted with a wholebody 1.5 tesla MR scanner (Horizon; General Electric Medical Systems, Milwaukee, WI). Images were obtained by using a single-shot, blipped, gradient-echo echoplanar pulse sequence using the parameters as follows: repetition time $(\mathrm{TR})=1000 \mathrm{msec}$, echo time $(\mathrm{TE})=43 \mathrm{msec}$, flip angle $(\mathrm{FA})=60^{\circ}$, slice thickness $=5 \mathrm{~mm}$, slice gap $=1 \mathrm{~mm}$, imaging matrix $=64 \times 64$, and field of view $($ FOV $)=24 \times 24 \mathrm{~cm}$. Based on our previous studies (Terada et al., 1995; Toma et al., 1997; Yazawa et al., 1998), we focused on the activity in the M1 and SMA, both of which may play a role in motor inhibition, and five axial slices were selected to cover these areas. One imaging session consisted of 60 time point dynamic scans (i.e., $60 \mathrm{sec}$ ). Before the actual imaging, the high resolution, three dimensional (3-D), anatomic T1-weighted images of the whole brain were collected $(\mathrm{TR}=10.8 \mathrm{msec}, \mathrm{TE}=1.8 \mathrm{msec}$, inversion time $(\mathrm{TI})=300$ msec, $\mathrm{FA}=15^{\circ}$, slice thickness $=1.5 \mathrm{~mm}$, no slice gap, imaging matrix $=$ $256 \times 256$, and FOV $=24 \times 24 \mathrm{~cm}$ ). Additional anatomic T1-weighted images corresponding to the echoplanar images of five slices were also obtained $\left(\mathrm{TR}=600 \mathrm{msec}, \mathrm{TE}=17 \mathrm{msec}, \mathrm{FA}=30^{\circ}\right.$, slice thickness $=5$ $\mathrm{mm}$, slice gap $=1 \mathrm{~mm}$, imaging matrix $=256 \times 256$, and $\mathrm{FOV}=24 \times$ $24 \mathrm{~cm})$.

Data analysis. Images were analyzed using SPM 96 software (Wellcome Department of Cognitive Neurology, London, UK), and calculations and image matrix manipulations were performed in Matlab (Mathworks, Sherborn, MA) on a Sun Sparc Ultra 2 workstation (Sun Microsystems, Mountain View, CA). The initial nine scans in each session were excluded from the analysis because of the nonequilibrium state of magnetization. The effect of head motion across scans was corrected by realigning all the scans to the first one, using a least sum of squares method with three-dimensional sinc interpolation (Friston et al., 1994). Because each motor trial was performed in a self-initiated manner, the onset of motor trial relative to the sequential number of scans within each session was variable among trials. For each task, therefore, all the series of dynamic scans were realigned time-locked to the motor trial. As a result, either the first or the last several scans were not included in the analysis, depending on the session. Global normalization was 
Box-car functions

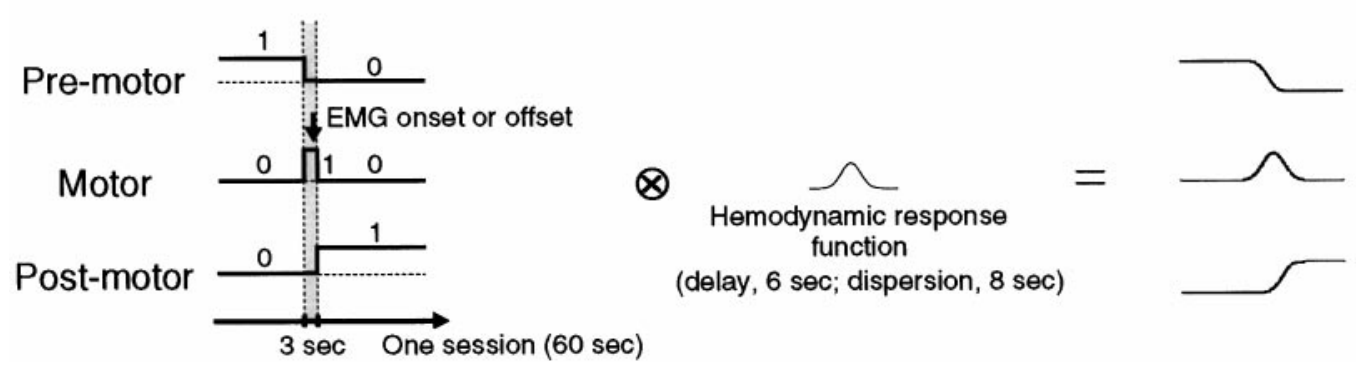

Figure 2. Three box-car functions used in the present analysis for representing premotor, motor, and postmotor phases. Motor phase contains three scans: one coinciding with the EMG onset or offset and the preceding two. Each time series is convolved with a Gaussian-shaped hemodynamic response function to produce three regressors (expected hemodynamic responses). $\otimes$ denotes convolution.

performed by linearly scaling the activity in each pixel with respect to the global activity. Data were smoothed in a spatial domain (full width at half-maximum $=3.75 \mathrm{~mm}$ ) to improve the signal-to-noise ratio.

fMRI time series data were analyzed using a general linear model (Friston et al., 1995a). The analysis was performed for each task separately on an individual subject basis. Three box-car functions were constructed to model premotor, motor, and postmotor phases (Fig. 2, left). For each function, the value " 1 " was given for the phase of interest and " 0 " (zero) for the remaining phases. In the box-car function for the motor phase, one scan coinciding with the event onset and the two preceding scans ( $3 \mathrm{sec}$ altogether) were assigned 1 because the previous electrophysiological findings suggested that the cortex prepares for the muscle relaxation or contraction $2-3 \mathrm{sec}$ before the movement onset (Terada et al., 1995; Nagamine et al., 1996; Toma et al., 1997). Each box-car function was convolved with a Gaussian-shaped hemodynamic response function (delay, $6 \mathrm{sec}$; dispersion, $8 \mathrm{sec}$ ) (Friston et al., 1995b; Worsley and Friston, 1995) to produce three regressors of interest used in the analysis (Fig. 2, right). Systematic difference across trials was modeled as a confounding effect. The general linear model calculated a weighting coefficient for each regressor. To focus on a transient signal change associated with motor trial, we calculated $t$ deviates at each voxel by using a linear contrast of $[-1,2,-1]$ for [premotor, motor, postmotor] and, after transforming into $Z$ scores with the unit normal distribution, created SPM $\{Z\}$ maps consisting of the voxels with $Z>3.09$ and $p<$ 0.05 with correction for multiple comparisons. In the present report, we use the term "activation" to represent the transient signal increase disclosed by the above analysis for the sake of convenience.

First, the locus of activated foci was visually inspected by superimposing SPM $\{Z\}$ onto both the anatomical and echoplanar images. To obtain the precise anatomical information, especially to disclose the anatomical relationship between the activation on the medial frontal wall and the vertical line passing through the anterior commissure (VAC line) in Talairach coordinates (Talairach and Tournoux, 1988), SPM $\{Z\}$ was transformed into the standardized Talairach space by applying the parameters obtained from the anatomical normalization of the 3-D anatomic image after coregistering it with five-slice functional and anatomic images. The $x, y$, and $z$ coordinates of the voxel with maximum $Z$ score in each region were statistically analyzed among the four tasks using multiple ANOVA (MANOVA).

For each activated brain area, signal intensity at the voxel with maximum $Z$ score was averaged across 10 sessions for each task. The baseline was defined as the first $10 \%$ of each session. The percent increase of the peak signal intensity was calculated with respect to the baseline. Mean time course of the signal change in the contralateral (left) M1 and SMA was obtained by averaging the signals across the subjects who showed the significant activation in each area. The difference of percent signal increase in each region was statistically examined by a two by two factorial ANOVA, with the factors of mode (relaxation vs contraction) and condition (movement vs isometric).

The region of interest (ROI) was set up on the left M1 and bilateral SMAs. By considering the possibility of a specific role of the SMA in the negative motor phenomenon (Lüders et al., 1995), the ROI over the SMA was further subdivided into the rostral (pre-SMA) and caudal (SMA proper) parts. Based on the previous review article on the human
PET studies (Picard and Strick, 1996), the pre-SMA was defined as $-12<x<12,0<y<30$, and $45<z<70$, and the SMA proper as $-12<x<12,-25<y<0$, and $40<z<70$ on the Talairach coordinates. The ROI for the left M1 was defined as a $30 \mathrm{~mm}$ cube, whose center corresponded to the mean location of the peak activation in the M1 across the tasks within each subject. The volume of these ROIs was $27 \mathrm{ml}$ for the M1 and $18 \mathrm{ml}$ for both the pre-SMA and SMA proper. Two by two factorial ANOVAs were conducted on the volume of the activated voxels in these areas.

\section{RESULTS}

\section{Task performance}

The onset of motor trial was $27.3 \pm 1.4 \mathrm{sec}($ mean $\pm \mathrm{SD})$ for the R_mv, $27.3 \pm 1.3 \mathrm{sec}$ for the C_mv, $26.4 \pm 1.5 \mathrm{sec}$ for the $\mathrm{R}_{-}$iso, and $25.8 \pm 1.6 \mathrm{sec}$ for the $\mathrm{C}_{-} \overline{\mathrm{i}}$ so. In the $\mathrm{R} \_\mathrm{mv}$, some subjects reported difficulty in avoiding the $\mathrm{FCU}$ contraction at the time of the relaxation of wrist extensors, whereas all the subjects found the $\mathrm{R}_{-}$iso easy to perform.

\section{Activated areas in voluntary muscle relaxation}

Despite the fact that the muscle contraction terminated at the time of motor trial in the $\mathrm{R}_{-} \mathrm{mv}$ and $\mathrm{R}$ _iso, the signal in the contralateral (left) M1 as well as in the bilateral SMAs increased transiently time-locked to the EMG offset. The left perirolandic activation was located exclusively on the precentral gyrus in all the subjects except for three who demonstrated additional peak activation in the primary sensory area $\left(\mathrm{C}_{-} \mathrm{mv}\right.$ and $\mathrm{R}_{-}$iso in two subjects, and $\mathrm{C}_{-}$iso in one).

Activated areas associated with the $\mathrm{R}_{-}$iso and $\mathrm{C}_{-}$iso in one representative subject are superimposed on his own anatomic MRI in Figure $3 A$. The robust activation was observed in the left M1 and bilateral SMAs during the $\mathrm{R}$ _iso. The same areas were activated also in the $\mathrm{C}_{-}$iso. In addition, the $\mathrm{R}$ iso showed activation in the bilateral dorsal premotor areas (PMd). Likewise, the $\mathrm{C}_{-}$iso demonstrated additional activation in the bilateral PMd and parietal areas. In Figure $3 B$, the mean signal changes across 10 sessions in the left M1 and SMA for the R_iso from the same subject are shown with solid lines. It is noteworthy that the signal changes were evident even in a single trial, which are shown by dots representing each single value at each sampling point, adjusted to the EMG offset.

\section{Activated areas and hemodynamic responses in the four tasks}

Table 1 presents the brain regions, showing the significant activation for each task and the maximum $\mathrm{Z}$ score, percent signal 
A

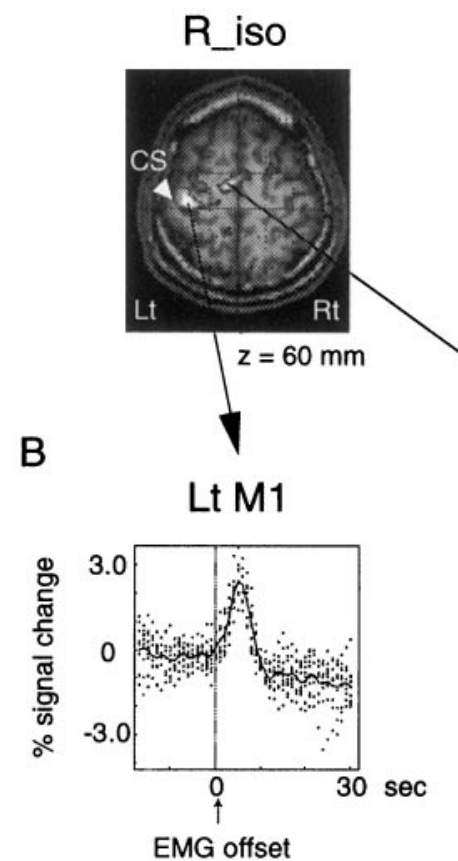

EMG offset
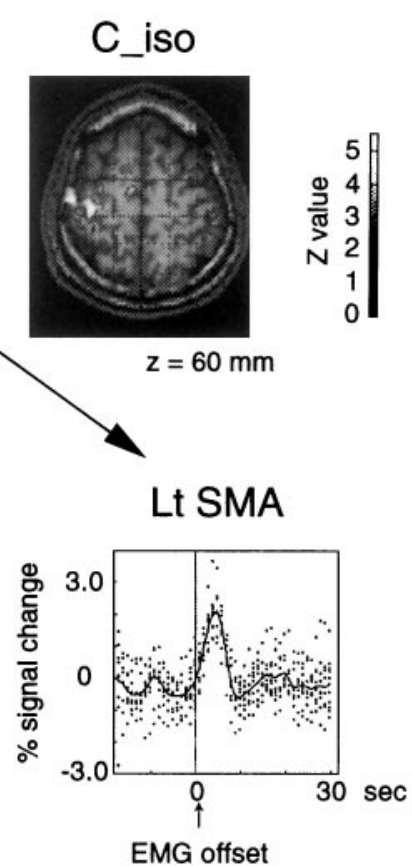

Figure 3. Activated areas for the isometric muscle relaxation $(R$ iso $)$ and contraction ( $C$ iso $)$ of the right hand $(A)$ and time course of signal change in the contralateral primary motor area $(L t M 1)$ and supplementary motor area $(L t S M A)$ for the $\mathrm{R}$ iso $(B)$, obtained from a single subject. $A$, Activated areas, which showed a significant transient increase of activity time-locked to the motor trial, are superimposed on the subject's own anatomic MRI. Brighter gray color represents higher $Z$ score. The illustrated slice corresponds to an axial plane of $z=60 \mathrm{~mm}$ in the standard space (Talairach and Tournoux, 1988). Activation is observed in the left M1 and bilateral SMAs for both the $\mathrm{R}_{-}$iso and $\mathrm{C}_{-}$iso. $C S$, Central sulcus. $B$, Averaged signals across 10 sessions at the voxel showing the maximum $Z$ score in the left M1 and SMA are represented by solid lines. Each $d o t$ represents data from a single trial at each sampling point. The vertical line indicates the offset of EMG activity. Clear transient increase of activity is observed even in a single trial and is highly reproducible.

increase, and the number of subjects for each region. For both the $\mathrm{R}$ _iso and $\mathrm{C}$ _iso, the left M1 and bilateral SMAs were activated in all eight subjects. By contrast, the activation was less consistent for both the R_mv and C_mv: five for the left M1 and seven for the bilateral SMAs. The two factorial ANOVA revealed that the percent signal increase in these three areas was not statistically different between the relaxation and contraction modes (left M1, $F_{(1,20)}=0.94, p=0.34$; left SMA, $F_{(1,20)}=0.44, p=0.51$; right SMA, $\left.F_{(1,20)}=0.28, p=0.60\right)$ or between the movement and isometric conditions (left M1, $F_{(1,20)}=0.10, p=0.75$; left SMA, $F_{(1,20)}=0.054, p=0.82$; right SMA, $\left.F_{(1,20)}=0.87, p=0.36\right)$. There was no interaction between the mode and condition (left $\mathrm{M} 1, F_{(1,20)}=0.61, p=0.44$; left SMA, $F_{(1,20)}=0.73, p=0.40$; right SMA, $\left.F_{(1,20)}=0.50, p=0.49\right)$.

In Table 2, the mean coordinates of the peak activation in the left M1 and bilateral SMAs are presented for each task. The MANOVA showed no significant difference in the location of activation for the left M1 (Wilks' lambda $=0.46 ; p=0.89$ ) or bilateral SMAs (Wilks' lambda $=0.70 ; p=0.71$ ) among the tasks.

Figure $4 A$ summarizes the commonly activated areas among subjects for each task. Only the pixels showing significant activation in three or more subjects are illustrated. Three motor-related

\begin{tabular}{|c|c|c|c|}
\hline $\begin{array}{l}\text { Region } \\
\text { (Brodmann's area) }\end{array}$ & $\begin{array}{l}\text { Maximum } \\
Z\end{array}$ & $\begin{array}{l}\% \text { signal } \\
\text { increase }\end{array}$ & $\begin{array}{l}\text { Number } \\
\text { of subjects }\end{array}$ \\
\hline \multicolumn{4}{|l|}{ R_mv } \\
\hline \multicolumn{4}{|l|}{ Left } \\
\hline M1 (4) & $6.4 \pm 1.1$ & $2.2 \pm 1.5$ & 5 \\
\hline SMA (6) & $6.1 \pm 1.0$ & $1.5 \pm 0.6$ & 7 \\
\hline PMd (6) & $5.4 \pm 1.6$ & $1.0 \pm 0.6$ & 3 \\
\hline LPi (40) & $5.7 \pm 0.3$ & $3.5 \pm 4.0$ & 4 \\
\hline \multicolumn{4}{|l|}{ Right } \\
\hline SMA (6) & $6.8 \pm 0.7$ & $1.7 \pm 0.9$ & 7 \\
\hline PMd (6) & $6.1 \pm 0.9$ & $1.2 \pm 0.4$ & 3 \\
\hline LPi (40) & $4.5 \pm 0.4$ & $1.7 \pm 1.4$ & 3 \\
\hline \multicolumn{4}{|l|}{ C_mv } \\
\hline \multicolumn{4}{|l|}{ Left } \\
\hline M1 (4) & $6.5 \pm 0.8$ & $2.3 \pm 1.4$ & 5 \\
\hline SMA (6) & $6.2 \pm 1.1$ & $1.7 \pm 0.6$ & 7 \\
\hline PMd (6) & $5.6 \pm 1.4$ & $1.6 \pm 0.9$ & 4 \\
\hline LPi (40) & $6.2 \pm 1.2$ & $3.8 \pm 2.6$ & 5 \\
\hline \multicolumn{4}{|l|}{ Right } \\
\hline SMA (6) & $5.7 \pm 0.8$ & $1.8 \pm 0.9$ & 7 \\
\hline PMd (6) & $5.6 \pm 0.5$ & $1.7 \pm 0.7$ & 5 \\
\hline LPi (40) & $5.3 \pm 1.0$ & $3.0 \pm 1.8$ & 4 \\
\hline \multicolumn{4}{|l|}{$\mathrm{R}$ _iso } \\
\hline \multicolumn{4}{|l|}{ Left } \\
\hline M1 (4) & $7.0 \pm 0.8$ & $2.0 \pm 0.5$ & 8 \\
\hline SMA (6) & $6.4 \pm 1.1$ & $1.4 \pm 0.5$ & 8 \\
\hline PMd (6) & $5.9 \pm 0.7$ & $1.0 \pm 0.7$ & 5 \\
\hline LPi (40) & $4.9 \pm 0.3$ & $2.0 \pm 1.1$ & 4 \\
\hline \multicolumn{4}{|l|}{ Right } \\
\hline SMA (6) & $6.7 \pm 0.9$ & $1.8 \pm 0.8$ & 8 \\
\hline PMd (6) & $5.5 \pm 0.9$ & $1.3 \pm 0.5$ & 5 \\
\hline LPi (40) & $5.1 \pm 0.1$ & $1.5 \pm 0.4$ & 2 \\
\hline \multicolumn{4}{|l|}{$\mathrm{C}_{-}$iso } \\
\hline \multicolumn{4}{|l|}{ Left } \\
\hline M1 (4) & $6.2 \pm 1.0$ & $3.2 \pm 2.1$ & 8 \\
\hline SMA (6) & $6.1 \pm 0.4$ & $1.8 \pm 0.7$ & 8 \\
\hline PMd (6) & $6.1 \pm 0.4$ & $1.9 \pm 0.9$ & 3 \\
\hline LPi (40) & $5.2 \pm 0.4$ & $2.7 \pm 1.2$ & 4 \\
\hline \multicolumn{4}{|l|}{ Right } \\
\hline SMA (6) & $6.0 \pm 0.8$ & $1.8 \pm 0.8$ & 8 \\
\hline PMd (6) & $5.4 \pm 1.2$ & $1.7 \pm 1.3$ & 4 \\
\hline LPi (40) & $4.5 \pm 0.6$ & $2.8 \pm 2.0$ & 4 \\
\hline
\end{tabular}

R_mv, Muscle relaxation with movement; C_mv, muscle contraction with movement; $\mathrm{R}$ iso, isometric muscle relaxation; $\mathrm{C}$ iso, isometric muscle contraction; $\mathrm{M} 1$, primary motor area; SMA, supplementary motor area; PMd, dorsal premotor area; $\mathrm{LPi}$, inferior parietal area.

cortices, the left M1 and bilateral SMAs, were activated commonly in the four tasks. Figure $4 B$ compares the time course of the hemodynamic response in each area among the four tasks. The peak time of the observed fMRI signal was similar in the four tasks, peaking at $\sim 5 \mathrm{sec}$ after the motor trial. In all the tasks, increase of the hemodynamic response in the left M1 and SMA was observed before the onset of motor trial (Fig. 4B). As for the postmotor phase, the response in the left M1 remained elevated only in the $\mathrm{C}_{-}$iso. The $\mathrm{C}_{-}$iso required sustained maximal muscle contraction throughout the postmotor phase, whereas, in the C_mv, the subject kept the wrist extension only against the gravity with almost the same muscle force as that during the premotor phase. 


\begin{tabular}{|c|c|c|c|}
\hline & $x$ & $y$ & $z$ \\
\hline \multicolumn{4}{|l|}{ Lt M1 } \\
\hline $\mathrm{R} \_\mathrm{mv}(n=5)$ & $-34.4 \pm 2.6$ & $-15.2 \pm 3.0$ & $54.8 \pm 6.3$ \\
\hline $\mathrm{C}_{-} \mathrm{mv}(n=5)$ & $-34.8 \pm 2.7$ & $-16.0 \pm 2.0$ & $54.8 \pm 6.7$ \\
\hline $\mathrm{R}$ iso $(n=8)$ & $-35.8 \pm 2.9$ & $-15.5 \pm 3.5$ & $59.0 \pm 6.3$ \\
\hline $\mathrm{C}_{-}$iso $(n=8)$ & $-35.0 \pm 4.4$ & $-16.3 \pm 3.9$ & $58.3 \pm 7.9$ \\
\hline \multicolumn{4}{|l|}{ SMA } \\
\hline R_mv $(n=7)$ & $2.6 \pm 3.2$ & $3.4 \pm 4.3$ & $55.4 \pm 9.4$ \\
\hline C_mv $(n=7)$ & $1.1 \pm 1.6$ & $2.0 \pm 3.7$ & $54.6 \pm 10$ \\
\hline $\mathrm{R}_{-}$iso $(n=8)$ & $1.3 \pm 5.3$ & $3.3 \pm 5.8$ & $51.3 \pm 7.6$ \\
\hline $\mathrm{C}_{-}$iso $(n=8)$ & $-1.5 \pm 3.9$ & $3.8 \pm 6.9$ & $51.5 \pm 9.9$ \\
\hline
\end{tabular}

Coordinates showing the highest $Z$ score were compared in the left M1 as well as in the bilateral SMAs.

R_mv, Muscle relaxation with movement; $\mathrm{C}_{-} \mathrm{mv}$, muscle contraction with movement; R_iso, isometric muscle relaxation; C_iso, isometric muscle contraction.

We found an additional activation in other cortical areas in some subjects including the PMd and inferior parietal area (Table 1). These areas, however, were less commonly activated compared with the left M1 and bilateral SMAs.

\section{Comparison of the activated volume among the four tasks}

After visual inspection of each individual data, we found that the activated area on the medial frontal wall was more extensive in the muscle relaxation than in the muscle contraction. Figure 5 shows a typical example of the activated area on the medial frontal wall in a single subject. The volume of voxels that showed significant activation within each ROI was compared among the four tasks (Fig. 6). The two factorial ANOVA revealed that the activated volume was significantly larger for the relaxation mode than for the contraction mode in the pre-SMA $\left(F_{(1,28)}=6.1 ; p=\right.$ $0.020)$ as well as in the SMA proper $\left(F_{(1,28)}=4.7 ; p=0.039\right)$, but no difference was found between the movement and isometric conditions (pre-SMA, $F_{(1,28)}=0.23, p=0.63$; SMA proper, $\left.F_{(1,28)}=0.51, p=0.48\right)$. There was no interaction between the mode and condition (pre-SMA, $F_{(1,28)}=0.51, p=0.48$; SMA proper, $\left.F_{(1,28)}=1.1, p=0.31\right)$. By contrast, the left M1 showed no difference between the relaxation and contraction modes $\left(F_{(1,28)}=0.075, p=0.79\right)$, whereas the activated volume was significantly larger for the isometric condition than for the movement condition (main effect of condition, $F_{(1,28)}=5.9, p=0.022$ ). There was no interaction between the mode and condition in the left M1 either $\left(F_{(1,28)}=0.74 ; p=0.40\right)$.

\section{DISCUSSION}

In the present study with the time resolution of $1 \mathrm{sec}$, the transient fMRI signal increase time-locked to the beginning of voluntary muscle relaxation was observed in the contralateral M1 and bilateral SMAs. The similar transient change of activity in the same areas was evident also during voluntary muscle contraction. The present finding showing the activation of the same cortical areas for both the muscle relaxation and contraction is in conformity with the previous electrophysiological study showing the similar distribution of BP over the head in the two motor modes (Terada et al., 1995). Absence of significant difference in the location of the peak activation in either the contralateral M1 or bilateral SMAs between the muscle relaxation and contraction suggests that motor inhibitory area, if present within these areas, is actually interspersed with the excitatory zone or present in its close vicinity.

In the interpretation of the present result, the effect of sensory feedback needs to be considered. The afferent feedback from movement execution may cause the perirolandic activation, as reported in the studies of EEG (Mima et al., 1996) and PET (Weiller et al., 1996) using passive movements. In the present study, however, the peak activation around the rolandic area was located anterior to the central sulcus in the majority of subjects. Furthermore, the contralateral M1 was more clearly activated in the $\mathrm{R}$ _iso (with little proprioceptive inputs) than in the $\mathrm{R}$-mv (with distinct joint movement). The fact that the increase of hemodynamic response was observed during the premotor phase in the contralateral M1 gives further supportive evidence that the motor preparation or execution caused the main activation over the rolandic area. Based on these findings, it is unlikely that the afferent feedback is the main contributor to the perirolandic activation observed in the present study. It is impossible to completely reject the possibility that other muscles whose EMGs were not monitored showed concomitant contraction with the relaxation of the targeted muscles. However, such an accidental muscle contraction, if any, is unlikely to produce greater activation than the muscle contraction that the subject explicitly performed.

As regards the temporal sequence of activation between the M1 and SMA, the previously reported data based on the subdural (Ikeda et al., 1992) and depth (Rektor et al., 1994) recordings of BP have been inconsistent. The finding of the present study was also unclear because of the limited time resolution of the eventrelated fMRI technique. The conclusion about the sequential cortical activation in the M1 and SMA awaits further supportive evidence from the studies using other techniques.

\section{Role of the M1 in motor inhibition}

A transient signal increase in the M1 associated with voluntary muscle relaxation suggests two possibilities in terms of the types of activated neurons: corticospinal projection neurons targeting spinal inhibitory interneurons and intrinsic inhibitory interneurons.

Studies in monkeys using the stimulus- or spike-triggered averaging technique showed that pure suppression of the muscle contraction with no facilitation of the antagonists occurred after corticomotoneuronal firing, possibly through the spinal inhibitory interneurons (Cheney et al., 1985; Lemon et al., 1987). Therefore, this type of disynaptic active inhibition of the spinal motoneurons is one possible motor inhibitory mechanism. The scalp-recorded EEG is thought to represent summed synaptic potentials arising from a large population of corticospinal projection neurons, which are arrayed perpendicularly to the cortical surface (Daly and Pedley, 1990). Epileptic negative myoclonus, defined as an interruption of tonic muscle activity time-locked to the EEG spike without an antecedent positive myoclonus, was shown to involve the contralateral centroparietal cortical area (Tassinari et al., 1995). These findings suggest that the corticospinal projection neurons may participate in the active inhibition of the spinal motoneurons.

A pause of the tonic excitatory corticospinal volley to the spinal motoneurons, mediated by intracortical inhibitory interneurons, may be another possible motor inhibitory mechanism taking place within the M1. It was reported that the low-intensity transcranial magnetic stimulation (TMS) could elicit a pause of ongoing EMG activity without any preceding motor-evoked potential, possibly by activating the intracortical inhibitory interneurons (Inghilleri 


\section{A}

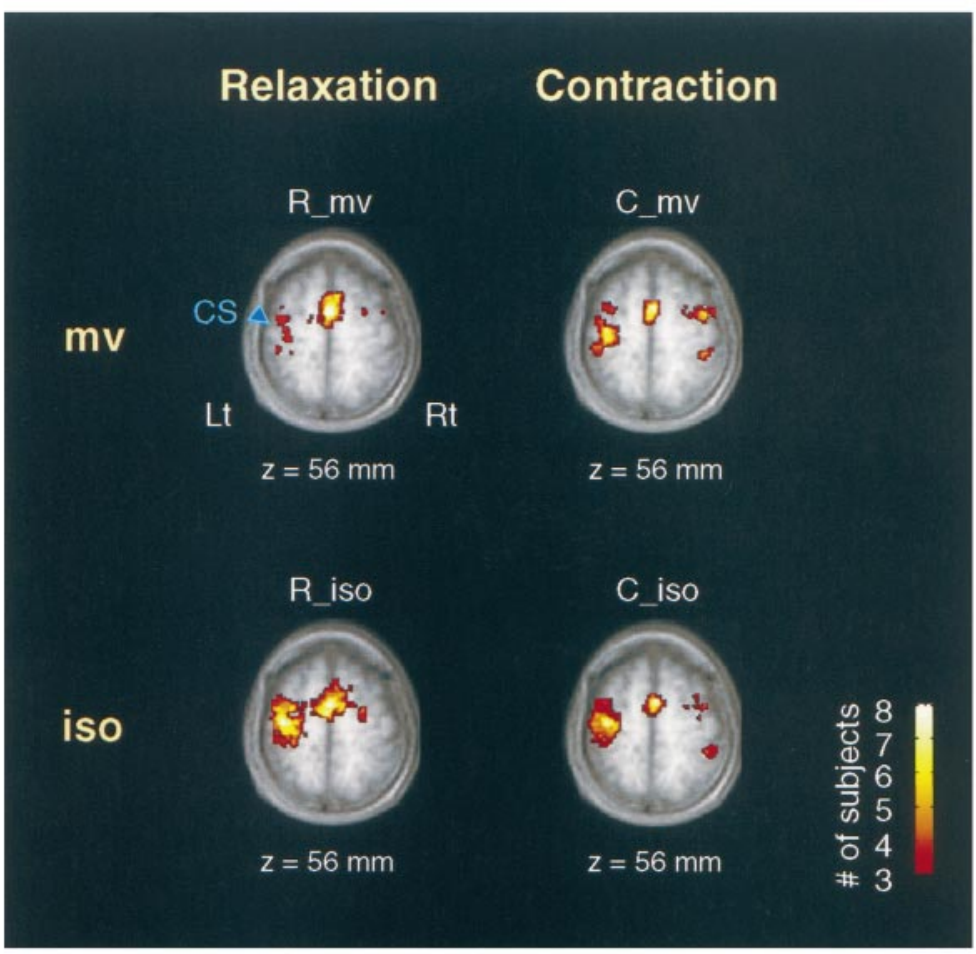

Figure 4. Activated areas $(A)$ and time course of the hemodynamic response $(B)$ in each task. $A$, Spatially normalized SPM $\{Z\}$ maps were superimposed across eight subjects on an axial plane $(z=56 \mathrm{~mm})$ of the averaged anatomic MRI from all the subjects. Note that this figure does not represent the direct statistical analysis across multiple subjects, instead the illustrated areas indicate the overlap in three or more of eight subjects. The right side of the brain is shown on the right side of the image. Brighter yellow color represents a larger number of subjects showing significant activation in the pixel. The majority of subjects show common activation in the contralateral (left) M1 and bilateral SMAs for the R_mv and $\mathrm{R}$ iso. $B$, Averaged signal changes at the local maxima in the left M1 and SMA across the subjects who showed significant activation during each task. Vertical interrupted lines represent the onset of motor trial. A similar signal change is observed in the four motor tasks in the left M1 and SMA. Note that the number of subjects is different among tasks and among regions.

B
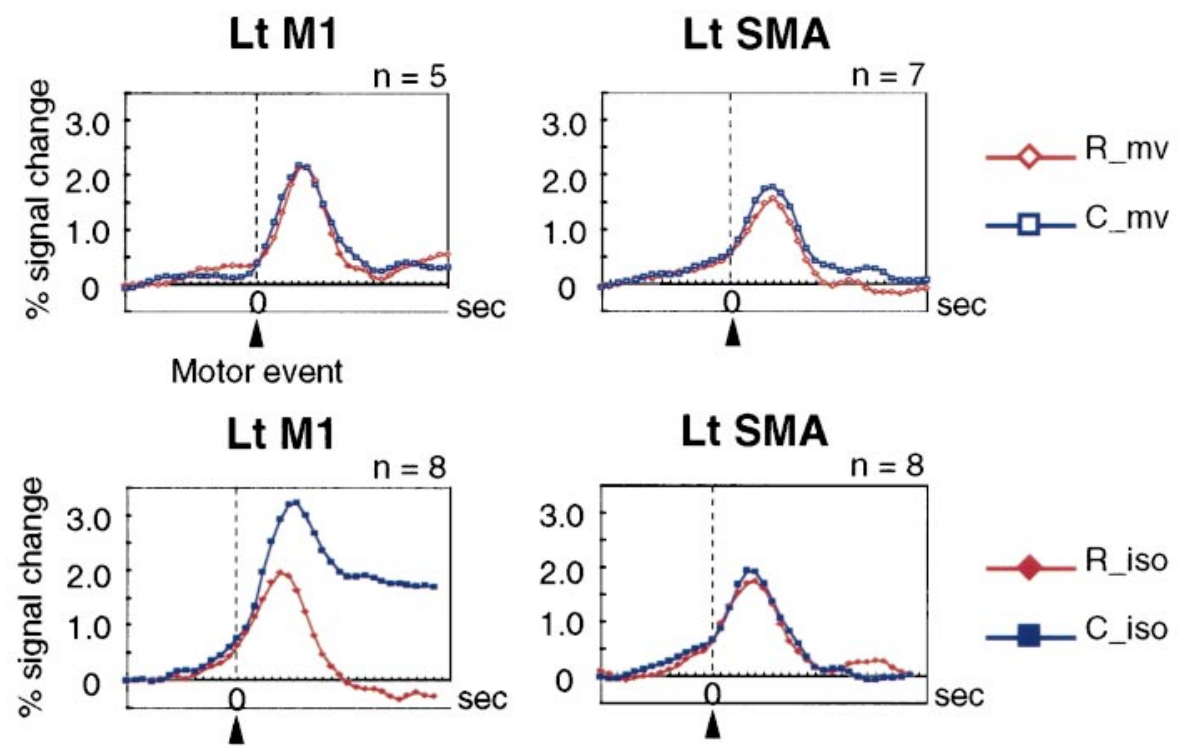

et al., 1993; Wassermann et al., 1993; Davey et al., 1994). Dystonia is a movement disorder characterized by abnormal cocontraction of agonist and antagonist muscles at rest and excessive contraction of irrelevant muscles during the movement execution (Mink, 1996). The double-pulse TMS, which tests the balance between the excitability of intracortical inhibitory and excitatory circuits, gave rise to abnormal result in patients with focal dystonia (Ridding et al., 1995), suggesting that the impairment of muscle relaxation in dystonia is partially caused by the impairment of intracortical inhibitory interneurons. These findings suggest that the excitation of intracortical inhibitory interneurons causing the withdrawal of ongoing input to the pyramidal neurons may participate in voluntary muscle relaxation.

Recently, Rothwell et al. (1998) reported that BP preceding the isometric muscle relaxation from a pinch was smaller in amplitude and less extensive than that preceding the isometric muscle contraction, particularly in the lateral leads overlying the M1. By contrast, no clear difference of $\mathrm{BP}$ was reported between the muscle relaxation and contraction with movement (Terada et al., 1995; Rothwell et al., 1998). Accordingly, two different motor inhibitory mechanisms are conceivable to explain the discrepant $\mathrm{BP}$ findings between these two kinds of muscle relaxation. 


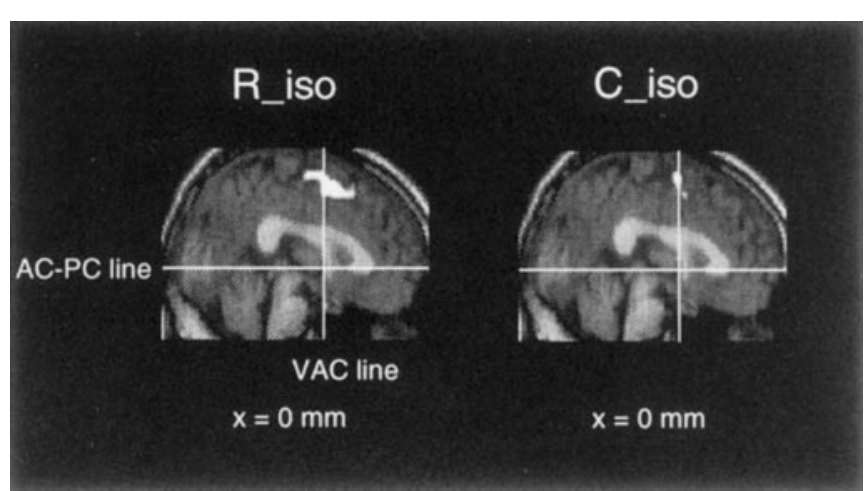

Figure 5. Activation on the medial frontal wall during the $\mathrm{R}$ iso and $\mathrm{C}_{-}$iso in a single subject. The illustrated slice corresponds to a sagittal plane of $x=0 \mathrm{~mm}$ in the standard space (Talairach and Tournoux, 1988). Activated area over the medial frontal wall is larger in the $\mathrm{R}_{-}$iso than in the $\mathrm{C}_{-}$iso.

Namely, muscle relaxation with movement may be achieved mainly by the excitation of descending corticospinal projections to produce active inhibition, whereas isometric muscle relaxation may be achieved mainly by the withdrawal of ongoing input to pyramidal neurons, mediated by the intracortical inhibitory neurons, which requires little preparation in motor cortex (Rothwell et al., 1998). Theoretically, intracortical inhibitory interneurons do not generate the potentials that can be detected from the scalp, because their dendrites spread in the various directions, thus canceling out the potentials generated by each individual neuron (Niedermeyer and Lopes da Silva, 1993). This is the most likely reason to explain the robust activation of M1 seen in the present $\mathrm{R}$ _iso task, which might have escaped the detection by the preceding EEG study. Alternatively, because of the delay and dispersion of the hemodynamic response, the different time course in $\mathrm{BP}$ between the $\mathrm{R}$ _iso and $\mathrm{R}$ _mv might not have been detected with the present fMRI technique. In fact, both of the two mechanisms, corticospinal projection neurons and intrinsic inhibitory interneurons, may function in the cortical process of motor inhibition in concert rather than exclusively from each other.

\section{Role of the SMA in motor inhibition}

Direct evidence for the participation of SMA in voluntary muscle relaxation was demonstrated by the study using subdural recordings in intractable epileptic patients during the presurgical evaluation, in which BP preceding voluntary muscle relaxation was recorded from the contralateral SMA (Yazawa et al., 1998).

Recently, the SMA is functionally subdivided into the rostral (pre-SMA) and caudal (SMA proper) parts in nonhuman primates (Tanji, 1994) as well as in humans (Picard and Strick, 1996). The SMA proper is directly connected to the M1 and spinal cord (Dum and Strick, 1992), and thus is thought to function either in parallel with or hierarchically superior to the M1. By contrast, the pre-SMA receives strong inputs from the prefrontal cortex and projects to the somatotopic representation of upper limb in the SMA proper, but lacks the direct connection to the M1 and spinal cord (Luppino et al., 1993). Therefore, it is supposed to play a superior role to the SMA proper. High-frequency electrical stimulation with subdural electrodes at the medial portion of the superior frontal gyrus anterior to the face area of SMA, probably corresponding to the pre-SMA, induced the termination of the sustained muscle contraction. This area was termed "supplementary negative motor area" by Lüders et al. (1995). The present
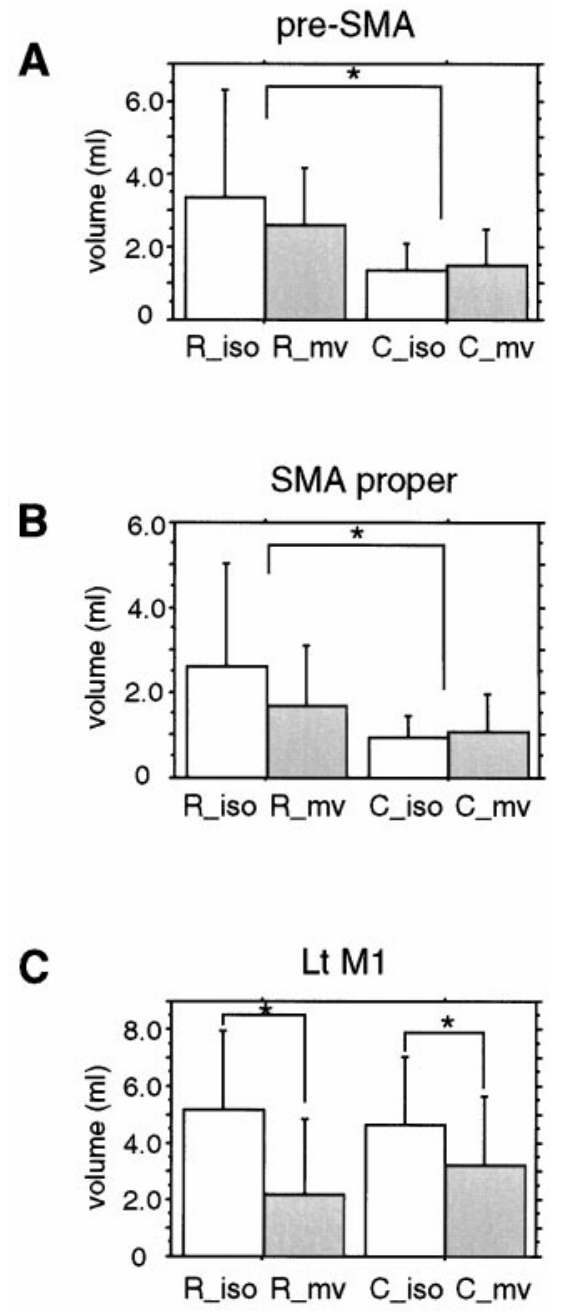

Figure 6. Volume of the activated areas during each task (mean $\pm \mathrm{SD}$ ) in the bilateral pre-SMA $(A)$, SMA proper $(B)$, and the contralateral (left) M1 $(C)$. The pre-SMA and SMA proper show significantly larger activation for the relaxation mode than for the contraction mode but no difference between the two conditions (i.e., movement vs isometric). By contrast, the activated volume in the left M1 is significantly larger for the isometric condition than for the movement condition, whereas there was no difference between the two modes (i.e., relaxation vs contraction). $* p<0.05$ by two-factors ANOVA.

study, however, showed significantly greater activation not only in the rostral but also in the caudal part of SMA for the muscle relaxation than for the muscle contraction, which suggests that both pre-SMA and SMA proper may play a role in motor inhibition. The question as to whether the regions responsible for higher motor control such as the pre-SMA actually act as "motor inhibitory center" or not still remains to be solved.

Alternatively, as suggested in the previous studies (Picard and Strick, 1996), the different activation in the pre-SMA between the muscle relaxation and contraction may be confounded by the fact that these two motor modes used in the present study were not of equal difficulty. As a matter of fact, in the R_mv, some subjects found it difficult to avoid the EMG activity of the wrist flexors when they tried to relax the wrist extensors. Accordingly, the activation in the pre-SMA may be partially caused by the relatively greater difficulty of the R_mv compared with the C_mv. In the isometric condition, however, all the subjects found the $\mathrm{R}$ _iso simple and easy to perform, as also reported by the previous study 
adopting the same task (Rothwell et al., 1998). It is, therefore, unlikely that the greater activation in the pre-SMA during the $\mathrm{R}$ iso as compared with the $\mathrm{C}_{-}$iso is caused by the difference in subjective difficulty.

By discriminating the signal associated with the muscle relaxation from that with the muscle contraction by the use of eventrelated fMRI technique, we clearly demonstrated that the activity in the M1 and SMA increased in association with the preparation and execution of voluntary muscle relaxation. It is postulated from the present findings that the abnormality of these neuronal substrates might be related to pathophysiology of certain movement disorders, especially dystonia (Ceballos-Baumann et al., 1995, 1997).

\section{REFERENCES}

Buckner RL, Bandettini PA, O'Craven KM, Savoy RL, Petersen SE, Raichle ME, Rosen B (1996) Detection of cortical activation during averaged single trials of a cognitive task using functional magnetic resonance imaging. Proc Natl Acad Sci USA 93:14878-14883.

Ceballos-Baumann AO, Passingham RE, Warner T, Playford ED, Marsden CD, Brooks DJ (1995) Overactive prefrontal and underactive motor cortical areas in idiopathic dystonia. Ann Neurol 37:2252-2257.

Ceballos-Baumann AO, Sheean G, Passingham RE, Marsden CD, Brooks DJ (1997) Botulinum toxin does not reverse the cortical dysfunction associated with writer's cramp. A PET study. Brain 120:571-582.

Cheney PD, Fetz EE, Sawyer-Palmer S (1985) Patterns of facilitation and suppression of antagonist forelimb muscles from motor cortex sites in the awake monkey. J Neurophysiol 53:805-820.

Daly DD, Pedley TA (1990) Current practice of clinical electroencephalography, Ed 2. New York: Raven.

Davey NJ, Romaiguère P, Maskill DW, Ellaway PH (1994) Suppression of voluntary motor activity revealed using transcranial magnetic stimulation of the motor cortex in man. J Physiol (Lond) 477:223-235.

Dum RP, Strick PL (1992) Medial wall motor areas and skeletomotor control. Curr Opin Neurobiol 2:836-839.

Friston KJ, Jezzard P, Turner R (1994) Analysis of functional MRI time-series. Hum Brain Mapp 1:153-171.

Friston KJ, Holmes AP, Worsley KJ, Poline J-P, Frith CD, Frackowiak RSJ (1995a) Statistical parametric maps in functional imaging: a general linear approach. Hum Brain Mapp 2:189-210.

Friston KJ, Holmes AP, Poline JB, Grasby PJ, Williams SC, Frackowiak RS, Turner R (1995b) Analysis of fMRI time-series revisited. NeuroImage 2:45-53.

Ikeda A, Lüders HO, Burgess RC, Shibasaki H (1992) Movementrelated potentials recorded from supplementary motor area and primary motor area. Brain 115:1017-1043.

Inghilleri M, Berardelli A, Cruccu G, Manfredi M (1993) Silent period evoked by transcranial stimulation of the human cortex and cervicomedullary junction. J Physiol (Lond) 466:521-534.

Jankowska E, Padel Y, Tanaka R (1976) Disynaptic inhibition of spinal motoneurons from the motor cortex in the monkey. J Physiol (Lond) 258:467-487.

Lemon RN, Muir RB, Mantel GWH (1987) The effects upon the activity of hand and forearm muscles of intracortical stimulation in the vicinity of corticomotor neurons in the conscious monkey. Exp Brain Res 66:621-637.

Lüders HO, Dinner DS, Morris HH, Wyllie E, Comair YG (1995) Cortical electrical stimulation in humans. The negative motor areas. Adv Neurol 67:115-129.

Luppino G, Matelli M, Camarda R, Rizzolatti G (1993) Corticocortical connections of area F3 (SMA proper) and area F6 (pre-SMA) in the macaque monkey. J Comp Neurol 338:114-140.

Mima T, Terada K, Maekawa M, Nagamine T, Ikeda A, Shibasaki H (1996) Somatosensory evoked potentials following proprioceptive stimulation of finger in man. Exp Brain Res 111:233-245.

Mink JW (1996) The basal ganglia: focused selection and inhibition of competing motor programs. Prog Neurobiol 50:381-425.
Nagamine T, Kajola M, Salmelin R, Shibasaki H, Hari R (1996) Movement-related slow cortical magnetic fields and changes of spontaneous MEG- and EEG- brain rhythms. Electroencephalogr Clin Neurophysiol 99:274-86.

Niedermeyer E, Lopes da Silva F (1993) Electroencephalography. basic principles, clinical applications, and related fields, Ed 3. Baltimore: Williams and Wilkins.

Picard N, Strick PL (1996) Motor areas of the medial wall: a review of their location and functional activation. Cereb Cortex 6:342-353.

Rektor I, Feve A, Buser P, Bathien N, Lamarche M (1994) Intracerebral recording of movement related readiness potentials: an exploration in epileptic patients. Electroencephalogr Clin Neurophysiol 90:273-283.

Ridding MC, Sheean G, Rothwell JC, Inzelberg R, Kujirai T (1995) Changes in the balance between motor cortical excitation and inhibition in focal, task specific dystonia. J Neurol Neurosurg Psychiatry 59:493-498.

Roland PE, Larsen B, Lassen NA, Skinhoj E (1980) Supplementary motor area and other cortical area in organization of voluntary movements in man. J Neurophysiol 43:137-150.

Rosen BR, Buckner RL, Dale AM (1998) Event-related functional MRI: past, present, and future. Proc Natl Acad Sci USA 95:773-780.

Rothwell JC, Higuchi K, Obeso JA (1998) The offset cortical potential: an electrical correlate of movement inhibition in man. Mov Disord 13:330-335.

Schmidt EM, McIntosh JS (1990) Microstimulation mapping of precentral cortex during trained movements. J Neurophysiol 64:1668-1682.

Shibasaki H, Sadato N, Lyshkow H, Yonekura Y, Honda M, Nagamine T, Suwazono S, Magata Y, Ikeda A, Miyazaki M, Fukuyama H, Asato R, Konishi J (1993) Both primary motor cortex and supplementary motor area play an important role in complex finger movement. Brain 116:1387-1398.

Shibasaki H, Ikeda A, Nagamine T, Mima T, Terada K, Nishitani N, Kanda M, Takano S, Hanazono T, Kohara N, Kaji R, Kimura J (1994) Cortical reflex negative myoclonus. Brain 117:477-486.

Talairach J, Tournoux P (1988) Co-planar stereotaxic atlas of the human brain. Stuttgart: Thieme.

Tanji J (1994) The supplementary motor area in the cerebral cortex. Neurosci Res 19:251-268.

Tassinari CA, Rubboli G, Parmeggiani L, Valzania F, Plasmati D, Riguzzi P, Michelucci R, Volpi L, Passarelli D, Meletti S, Fontana E, Bernardina BD (1995) Epileptic negative myoclonus. Adv Neurol 67:181-197.

Terada K, Ikeda I, Nagamine T, Shibasaki H (1995) Movement-related cortical potentials associated with voluntary muscle relaxation. Electroencephalogr Clin Neurophysiol 95:335-345.

Toma K, Terada K, Yazawa S, Ikeda A, Nagamine T, Shibasaki H (1997) Changes of magnetoencephalographic (MEG) rhythms associated with voluntary muscle relaxation. Electroencephalogr Clin Neurophysiol 103:155

Ugawa Y, Shimpo T, Mannen T (1989) Physiological analysis of asterixis: silent period locked averaging. J Neurol Neurosurg Psychiatry 52:89-93.

Wassermann EM, Pascual-Leone A, Valls-Solé J, Toro C, Cohen LG, Hallett M (1993) Topography of the inhibitory and excitatory responses to transcranial magnetic stimulation in a hand muscle. Electroencephalogr Clin Neurophysiol 89:424-433.

Weiller C, Jüptner M, Fellows S, Rijntjes M, Leonhardt G, Kiebel S, Müller S, Diener HC, Thilmann AF (1996) Brain representation of active and passive movements. NeuroImage 4:105-110.

Worsley KJ, Friston KJ (1995) Analysis of fMRI time-series revisited again. NeuroImage 2:173-181.

Yazawa S, Ikeda A, Kunieda T, Mima T, Nagamine T, Ohara S, Terada K, Taki W, Kimura J, Shibasaki H (1998) Human supplementary motor area is active in preparation for both voluntary muscle relaxation and contraction: subdural recording of Bereitschaftspotential. Neurosci Lett 244:145-148.

Young RR, Shahani BT (1986) Asterixis: one type of negative myoclonus. Adv Neurol 43:137-156. 